\title{
IQ SPECT Allows a Significant Reduction in Administered Dose and Acquisition Time for Myocardial Perfusion Imaging: Evidence from a Phantom Study
}

\author{
Federico Caobelli ${ }^{1,2}$, Stefano Ren Kaiser ${ }^{1}$, James T. Thackeray ${ }^{2}$, Frank M. Bengel ${ }^{2}$, Matteo Chieregato ${ }^{1}$, \\ Alberto Soffientini ${ }^{1}$, Claudio Pizzocaro ${ }^{1}$, Giordano Savelli ${ }^{1}$, Marco Galelli ${ }^{1}$, and Ugo Paolo Guerra $^{1}$ \\ ${ }^{1}$ Department of Nuclear Medicine, Fondazione Poliambulanza, Brescia, Italy; and ${ }^{2}$ Klinik für Nuklearmedizin, Medizinische \\ Hochschule Hannover, Hannover, Germany
}

We recently demonstrated in a clinical trial the ability of a new protocol, IQ SPECT, to acquire myocardial perfusion imaging (MPI) studies in a quarter of the time (12 s/view) of the standard protocol, with preserved diagnostic accuracy. We now aim to establish the lower limit of radioactivity that can be administered to patients and the minimum acquisition time in SPECT MPI using an IQ SPECT protocol, while preserving diagnostic accuracy. Methods: An anthropomorphic cardiac phantom was used to acquire clinical rest scans with a simulated in vivo distribution of $99 \mathrm{mTc}$-tetrofosmin at full dose $(740 \mathrm{MBq})$ and at doses equal to $50 \%, 25 \%$, and $18 \%$. For each dose, 2 sets of images were acquired, with and without a transmural defect (TD). Variable acquisition times were also used for each dose. We analyzed raw data and reconstructed images, including no correction and correction for attenuation (AC), for scatter (SC), or for both (ACSC). Images were evaluated qualitatively and quantitatively in order to assess left ventricle (LV) wall thickness (full width at half maximum of the medial sections), TD, and cavity contrast in the LV wall. Data were compared across different acquisition times within the same dose and across doses with the same acquisition time. Results: Images were visually scored as very-good quality except those acquired with $4 \mathrm{~s} / \mathrm{view}$ or less at $100 \%$ dose and $6 \mathrm{~s} / \mathrm{view}$ or less with $50 \%, 25 \%$, or $18 \%$ dose, due to falsepositive defects. LV wall thickness was not significantly different among all acquisitions. Cavity contrast remained unchanged within the same dose for all images and tended to be higher in $A C$ and ACSC images. TD contrast remained unchanged within the same dose for all images. In SC and no-correction images, contrast was constant for all doses. AC images had significantly higher TD contrast values, and ACSC images showed a drop in TD contrast for a $50 \%$ dose. Conclusion: IQ SPECT effectively preserved both image quality and quantitative measurements with reduced acquisition time or administered dose in a phantom study. These findings suggest that approximately one eighth of the time, compared with standard protocols with a full dose, or a lower dose at an acquisition time of $12 \mathrm{~s} /$ view can be applied in MPI without the loss of diagnostic accuracy.

\footnotetext{
Received May 23, 2014; revision accepted Oct. 27, 2014.

For correspondence contact: Federico Caobelli, Klinik für Nuklearmedizin, Medizinische Hochschule Hannover, Carl Neuberg Strasse, 1, 30625 Hannover, Germany.

E-mail: Caobelli.federico@mh-hannover.de

Published online Nov. 20, 2014.

COPYRIGHT (c) 2014 by the Society of Nuclear Medicine and Molecular Imaging, Inc.
}

Key Words: coronary artery disease; ${ }^{99 m T c-t e t r o f o s m i n ~ S P E C T / C T ; ~}$ IQ SPECT; low counts acquisition; myocardial perfusion imaging

J Nucl Med 2014; 55:2064-2070

DOI: $10.2967 /$ jnumed.114.143560

\section{O}

PECT myocardial perfusion imaging (MPI) has been extensively validated for the diagnosis and prognosis of cardiac disease and is currently largely recommended in national and international guidelines (1).

Growing interest in shortening scan time or in reducing administered dose has led to the development of new hardware and modern software packages with the aim of improving image quality and diagnostic accuracy comparable or even superior to standard protocol $(2,3)$.

In a clinical trial, we have recently validated a new protocol, IQ SPECT, characterized by the use of multifocal collimators with dedicated reconstruction software (4). The new IQ SPECT with multifocal collimators, which can be applied as a plug-in on multipurpose cameras, has a favorable performance regarding sensitivity and contrast-to-noise ratio when compared with conventional cameras equipped with low-energy and high-resolution collimators (5). The unique geometry of the collimator, with holes focused at the center and near-parallel at the edge, facilitates enhanced sensitivity for the cardiac region while permitting acquisition of untruncated projection data. In conjunction with a cardiocentric scan orbit at $28 \mathrm{~cm}$ to maximize regional magnification, this configuration provides a 4 -fold-higher sensitivity for the cardiac region than parallel-hole collimators (6). Because the collimator response is not only distance-dependent but also variable across its face, an accurate modeling of magnification and the system point-response function (PRF) is essential. As such, the 3-dimensional PRF is modeled from the autocorrelation of the hexagonal shapes of the collimator holes at the entry and exit points (6), and the collimator is programmed to automatically measure hole orientation, thus taking into account deviations from design specifications that are introduced in the casting process. This collimator setup allows the higher sensitivity to be taken advantage of without the poorer spatial resolution or inconsistent reconstructed data that occur when low doses or short acquisition times are used. Furthermore, the implementation of a proprietary conjugate-gradient iterative reconstruction algorithm incorporating measured values for gantry deflections, collimator-hole angles, and system PRF contributes to 


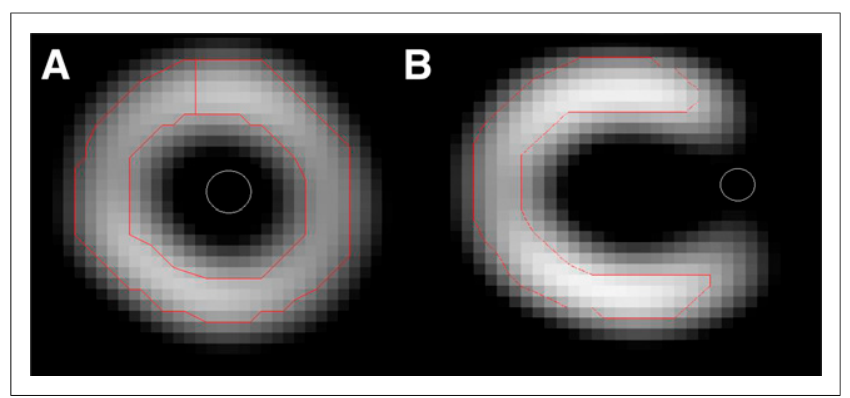

FIGURE 1. Example of ROls used for quantitative measurements in phantom study: contrast between LV wall (red borders) and LV cavity (white borders) (A) and contrast between LV wall (red borders) and TD (white borders) (B).

enhanced sensitivity and diagnostic accuracy (7). This algorithm also includes software for incorporating CT data into a patientspecific $\mu$ map and an energy window-based estimate to correct for patient-induced scatter to overcome poor count statistics. In fact, the addition of attenuation correction in the iterative algorithms improves the accuracy of the camera over resolution recovery alone and leads to greater consistency and more accurate reconstructed images (8). Taken together, these approaches maximize the image resolution and sensitivity under the constraint of shortened acquisition time. It remains unclear whether this maintained image quality also pertains to lower injected dose.

We therefore aimed to establish the lower limit of administered radioactivity and minimum acquisition time in SPECT MPI using the IQ SPECT system, while preserving diagnostic accuracy.

\section{MATERIALS AND METHODS}

\section{Phantom Preparation}

An anthropomorphic phantom featuring inserts to simulate lungs, liver, left ventricle (LV) wall, LV chamber, and a removable transmural perfusion defect (TD) (Torso Phantom and Cardiac Insert; Data Spectrum Corp.) was used in this study. The TD insert $(45 \times 2 \mathrm{~cm})$ was filled with nonradioactive water to simulate a TD and located in the mid to basal lateral position in the LV wall. The lung inserts were filled with Styrofoam (The Dow Chemical Co.) beads and nonradioactive water to simulate lung tissue attenuation density. The remaining chambers were filled with variable-activity concentrations of ${ }^{99 \mathrm{~m}} \mathrm{Tc}$ : $159 \pm 8 \mathrm{kBq} \times \mathrm{mL}^{-1}$ for the LV wall, $94 \pm 2 \mathrm{kBq} \times \mathrm{mL}^{-1}$ for the liver, and $7.6 \pm 0.2 \mathrm{kBq} \times \mathrm{mL}^{-1}$ for the chest and LV chamber. Concentrations were selected to reflect the in vivo distribution pattern of 740 $\mathrm{MBq}$ of ${ }^{99 \mathrm{~m}} \mathrm{Tc}$-tetrofosmin $(9,10)$.

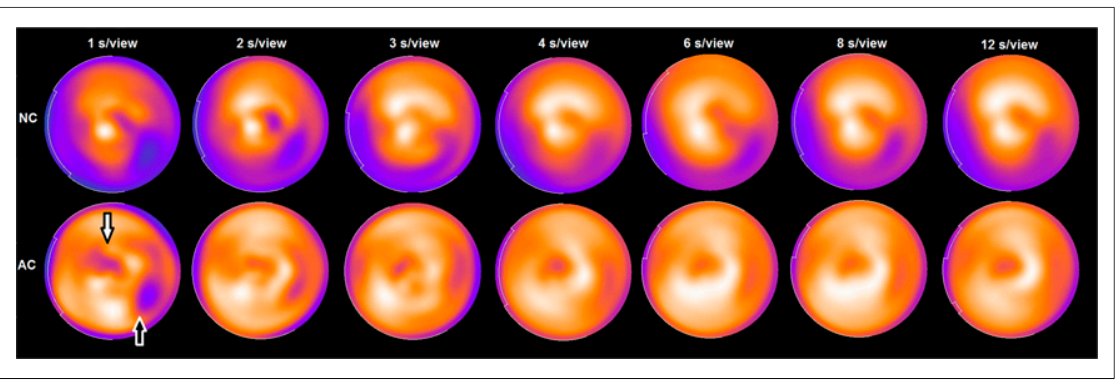

FIGURE 2. Polar plot images showing phantom image data (normal perfusion pattern) with no correction (NC) and attenuation correction (AC) applied. Images were obtained with $100 \%$ dose at different times (1, 2, 3, 4, 6, 8, and $12 \mathrm{~s} / \mathrm{view})$. Images obtained with at least $6 \mathrm{~s} / \mathrm{view}$ were scored as verygood quality. False-positive defects in anteroseptal (white arrow) and inferolateral wall (black arrow) were identified in images of lesser quality, denoted as minimal at 3-4 s/view and prominent on $1 \mathrm{~s} / \mathrm{view}$.
TABLE 1

LV Wall Thickness, Expressed as FWHM for Different Doses and Acquisition Times

\begin{tabular}{ccccc}
\hline & \multicolumn{4}{c}{ FWHM $(\mathrm{mm})$} \\
\cline { 2 - 5 } s/view & ACSC & AC & SC & NC \\
\hline $100 \%$ dose & & & \\
\hline 1 & $21.6 \pm 1.5$ & $21.4 \pm 1.8$ & $24.3 \pm 2.4$ & $25.0 \pm 1.7$ \\
2 & $20.3 \pm 0.4$ & $20.2 \pm 0.4$ & $21.6 \pm 0.4$ & $21.9 \pm 0.3$ \\
\hline 3 & $21.4 \pm 1.1$ & $21.5 \pm 1.0$ & $22.9 \pm 1.4$ & $23.0 \pm 1.2$ \\
\hline 4 & $21.0 \pm 0.5$ & $20.9 \pm 0.6$ & $22.3 \pm 0.7$ & $22.3 \pm 0.9$ \\
\hline 6 & $21.0 \pm 0.9$ & $21.1 \pm 0.9$ & $24.6 \pm 1.8$ & $24.1 \pm 1.5$ \\
\hline 8 & $22.3 \pm 0.9$ & $22.4 \pm 1.0$ & $24.5 \pm 1.6$ & $24.4 \pm 1.6$ \\
\hline 12 & $21.4 \pm 0.7$ & $21.3 \pm 1.0$ & $23.6 \pm 1.9$ & $22.8 \pm 1.6$ \\
\hline $50 \%$ dose & & & & \\
\hline 6 & $20.3 \pm 0.3$ & $20.2 \pm 0.4$ & $22.0 \pm 0.8$ & $22.3 \pm 1.0$ \\
\hline 12 & $21.4 \pm 1.1$ & $21.8 \pm 1.1$ & $24.1 \pm 1.7$ & $24.3 \pm 1.6$ \\
\hline $25 \%$ dose & & & & \\
6 & $21.0 \pm 0.3$ & $21.3 \pm 0.5$ & $22.8 \pm 0.4$ & $22.7 \pm 0.2$ \\
\hline 12 & $21.6 \pm 0.4$ & $21.0 \pm 0.2$ & $23.5 \pm 0.9$ & $23.5 \pm 1.5$ \\
\hline $18 \%$ dose & & & & \\
\hline 6 & $21.4 \pm 1.3$ & $21.8 \pm 1.5$ & $22.8 \pm 1.4$ & $23.0 \pm 1.6$ \\
\hline 12 & $21.8 \pm 0.2$ & $21.7 \pm 0.3$ & $24.8 \pm 0.6$ & $23.9 \pm 0.3$ \\
\hline 24 & $22.0 \pm 0.2$ & $22.1 \pm 0.8$ & $23.6 \pm 0.8$ & $23.7 \pm 1.3$ \\
\hline & & & & \\
\hline
\end{tabular}
SD.

\section{Image Protocol and Acquisition}

IQ SPECT System. Images were acquired on a hybrid dual-head SPECT/CT system (Symbia T; Siemens Medical Solutions AG) equipped with multifocal collimators (Smartzoom) of $128 \times 128$ matrix size and a zoom factor of 1 . Each collimator consists of 48,000 hexagonal holes (diameter, $1.9 \mathrm{~mm}$; length, $40 \mathrm{~mm}$ ). Spatial resolution is progressively increased from the camera surface to the cardiac sweet-spot at $28 \mathrm{~cm}$, which was set as the center of rotation for this study, thereby keeping the LV phantom at the highest magnification throughout the acquisition. A scanning arc of $104^{\circ}$ with $6^{\circ}$ angular steps was used for each of the camera heads. For each detector, 17 views were acquired. Acquired data were then reconstructed using a proprietary iterative reconstruction algorithm (3 iterations, 10 subsets) accounting for gantry deflections, collimator-hole angles, and system PRF (4). Attenuation correction was applied using a patient-dedicated low-dose CT-derived $\mu$ map. An energy window-based estimate was used to correct for scatter, identifying dual-energy windows (127-155 keV, primary; 99-126 keV, scatter).

Imaging Protocol. Phantom images with and without the TD insert were acquired serially at the time of loading $(0 \mathrm{~h})$ and after 6,12 , and 15 h to simulate a dose reduction to $50 \%, 25 \%$, and $18 \%$, respectively. This approach minimized variability in activity loading among different phantom cavities.

For each dose, different acquisition times were also used (for the 100\% dose, 1, 2, 3, 4, 6, 8, 


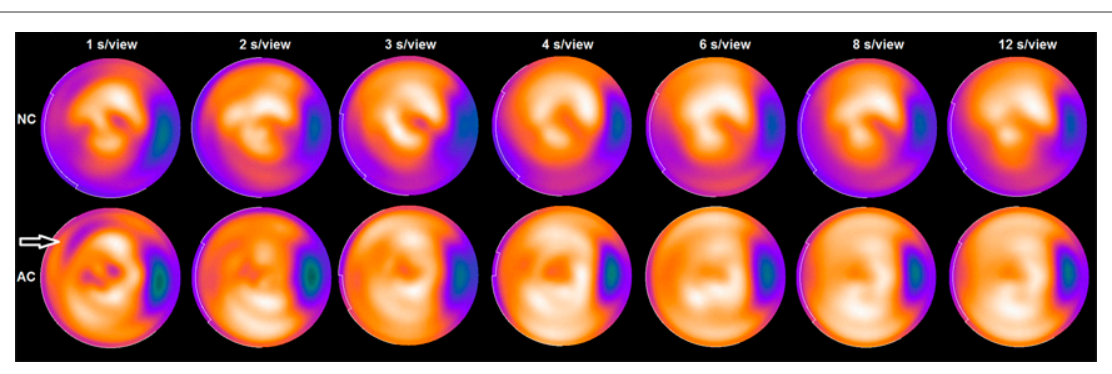

FIGURE 3. Polar plot images showing phantom image data (transmural lateral wall perfusion defect) with no correction (NC) and attenuation correction (AC) applied. Images were obtained with $100 \%$ dose at different times $(1,2,3,4,6,8$, and $12 \mathrm{~s} / \mathrm{view})$. Images obtained with at least 6 $\mathrm{s} /$ view were scored as very-good quality. False-positive defects in anteroseptal wall were identified with shorter acquisition times.

and $12 \mathrm{~s} / \mathrm{view}$; for the $50 \%$ dose, 6 and $12 \mathrm{~s} / \mathrm{view}$; for the $25 \%$ dose, 6 and $12 \mathrm{~s} / \mathrm{view}$; and for the $18 \%$ dose, 6, 12, and $24 \mathrm{~s} / \mathrm{view})$. Acquisitions were performed sequentially from shortest to longest time.

Images were analyzed with and without attenuation or scatter correction, generating 4 datasets: with no corrections (NC), attenuationcorrected (AC), scatter-corrected (SC), and attenuation- and scattercorrected (ACSC). The software for SC and AC was provided by the manufacturer using default settings. Alignment between CT and SPECT images was visually verified. Images have been qualitatively and quantitatively evaluated.

\section{Visual Analysis}

The quantitative perfusion SPECT-quantitative gated SPECT software and the dedicated IQ SPECT protocol generated left ventricular polar maps of perfusion, which were divided into 17 segments. Three experienced readers, masked to acquisition modality, reviewed the images and evaluated the map uniformity, the extent of TD, and the presence of unexpected artifacts. Uptake in each segment was scored using a 5-point score according to guidelines of the American Society of Nuclear Medicine (11) (normal perfusion, 0; mild reduction in counts not definitively abnormal, 1 ; moderate reduction in counts - definitively abnormal, 2; severe reduction in uptake, 3 ; and absent uptake, 4). Interobserver discrepancies were resolved by consensus.

\section{Quantitative Analysis}

After reconstruction, all image sets were processed to evaluate the following 3 parameters, associated with image quality for MPI: LV wall thickness, contrast between LV wall and LV cavity, and contrast between LV wall and TD. Wall thickness and wallto-cavity contrast were evaluated only without the TD insert in the LV wall. Measurements were obtained using ImageJ software (12) and repeated at least 3 times by an experienced operator to minimize measurement errors. Quantitative data were compared for variable shortened image acquisition times at a constant dose (i.e., 100\%) and for variable lower doses at a constant image acquisition time (i.e., $12 \mathrm{~s} / \mathrm{view})$.

LV Wall Thickness. LV wall thickness was calculated as full width at half maximum (FWHM) of the myocardial signal in the midventricular section of the LV short-axis by fitting the central count profile with a theoretic gaussian one. The image was summed over 3 consecutive

TABLE 2

Total Counts and Cavity Contrast Values for Different Doses and Acquisition Times

\begin{tabular}{|c|c|c|c|c|c|}
\hline \multirow[b]{2}{*}{ s/view } & \multirow[b]{2}{*}{ Total counts $\left(\times 10^{5}\right)$} & \multicolumn{4}{|c|}{ Cavity contrast (\%) } \\
\hline & & ACSC & $A C$ & SC & NC \\
\hline \multicolumn{6}{|c|}{$100 \%$ dose } \\
\hline 1 & 0.9 & $100.5 \pm 2.4$ & $100.9 \pm 2.2$ & $69.6 \pm 5.0$ & $73.0 \pm 5.5$ \\
\hline 2 & 1.9 & $98.1 \pm 3.0$ & $98.1 \pm 3.1$ & $81.3 \pm 4.7$ & $72.1 \pm 7.1$ \\
\hline 3 & 2.9 & $95.3 \pm 2.8$ & $92.9 \pm 4.8$ & $87.8 \pm 9.2$ & $87.5 \pm 4.1$ \\
\hline 4 & 3.6 & $94.8 \pm 3.5$ & $94.1 \pm 4.2$ & $76.0 \pm 5.3$ & $78.6 \pm 5.2$ \\
\hline 6 & 5.4 & $94.4 \pm 3.0$ & $93.7 \pm 3.7$ & $79.2 \pm 3.6$ & $77.4 \pm 5.0$ \\
\hline 8 & 7.5 & $96.2 \pm 2.2$ & $95.1 \pm 3.5$ & $81.5 \pm 4.3$ & $77.1 \pm 5.3$ \\
\hline 12 & 11.1 & $94.4 \pm 3.8$ & $96.0 \pm 3.4$ & $82.1 \pm 3.9$ & $77.2 \pm 5.9$ \\
\hline \multicolumn{6}{|c|}{$50 \%$ dose } \\
\hline 6 & 3.2 & $95.7 \pm 2.9$ & $95.9 \pm 2.9$ & $84.6 \pm 4.3$ & $82.2 \pm 5.7$ \\
\hline 12 & 6.5 & $96.5 \pm 3.3$ & $96.4 \pm 3.4$ & $77.6 \pm 4.5$ & $74.5 \pm 5.2$ \\
\hline \multicolumn{6}{|c|}{$25 \%$ dose } \\
\hline 6 & 1.5 & $94.9 \pm 3.3$ & $94.5 \pm 3.8$ & $83.6 \pm 4.6$ & $80.4 \pm 5.2$ \\
\hline 12 & 3.1 & $95.4 \pm 3.7$ & $94.3 \pm 3.6$ & $81.3 \pm 4.5$ & $81.8 \pm 7.4$ \\
\hline \multicolumn{6}{|l|}{$18 \%$ dose } \\
\hline 6 & 1.2 & $93.3 \pm 3.2$ & $94.8 \pm 2.8$ & $86.2 \pm 5.2$ & $85.5 \pm 3.9$ \\
\hline 12 & 2.5 & $95.3 \pm 2.9$ & $95.1 \pm 3.2$ & $93.4 \pm 2.9$ & $89.4 \pm 3.6$ \\
\hline 24 & 4.9 & $93.7 \pm 3.5$ & $93.1 \pm 3.2$ & $87.6 \pm 4.2$ & $87.7 \pm 3.8$ \\
\hline
\end{tabular}

Differences are not statistically significant. Values are mean \pm SD. 


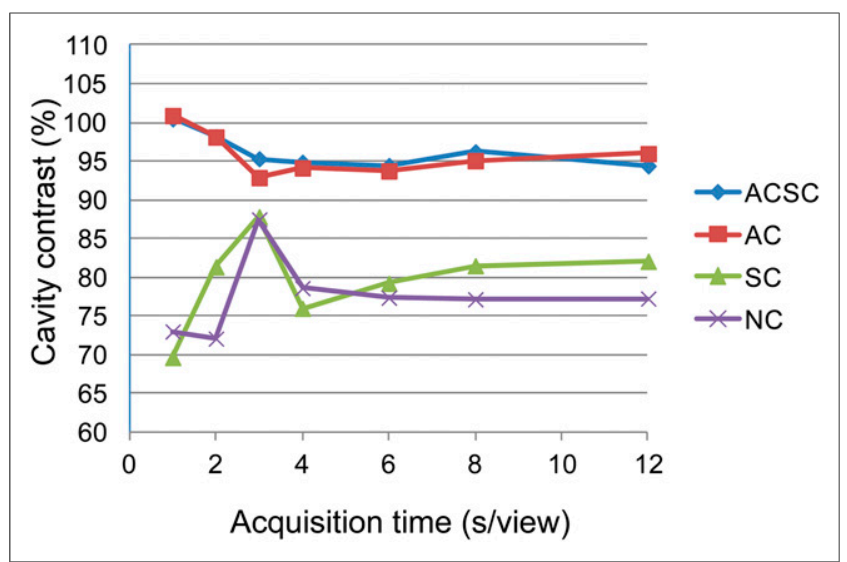

FIGURE 4. Cavity contrast at different acquisition times with constant dose $(100 \%)$. Cavity contrast remains unchanged for all acquisition times $(P=$ not significant).

slices in the midventricular section, and LV wall thickness was estimated as a mean of 3 different measurements taken from 3 different profiles - that is, anterior, septal, and inferior wall.

Cavity Contrast. To calculate the contrast between cavity and LV wall, 2 regions of interest (ROIs) were drawn on the midventricular short-axis images, to estimate the mean counts per voxel for the LV wall $\left(\eta_{\mathrm{LV}}\right)$ and ventricular chamber $\left(\eta_{\mathrm{IC}}\right)$, respectively. Figure 1A shows in detail how the measurements were performed. The dimensions of the circular ROI drawn on the ventricular cavity were kept identical among all acquired images (diameter, $9.6 \mathrm{~mm}$ ).

Cavity contrast was defined as:

$$
\left[\left(\eta_{\mathrm{LV}}-\eta_{\mathrm{IC}}\right) /\left(\eta_{\mathrm{LV}}+\eta_{\mathrm{IC}}\right)\right] /\left[\left(\mathrm{C}_{\mathrm{LV}}-\mathrm{C}_{\mathrm{IC}}\right) /\left(\mathrm{C}_{\mathrm{LV}}+\mathrm{C}_{\mathrm{IC}}\right)\right] \times 100,
$$

where $\mathrm{C}_{\mathrm{LV}}$ and $\mathrm{C}_{\mathrm{IC}}$ are the true tracer concentrations in the inserts simulating LV wall and LV cavity, respectively.

$T D$ Contrast. The contrast between TD and LV wall was evaluated in the LV short-axis by drawing ROIs on the central short-axis images of the phantom acquisitions with TD defect, to estimate the $\eta_{\mathrm{LV}}$ and TD $\left(\eta_{\text {TD }}\right)$, respectively. The dimension of the circular ROI drawn on TD was kept identical among all acquired images (diameter, $7.6 \mathrm{~mm}$ ) (Fig. 1B).

TD contrast was defined as:

$$
\left(\eta_{\mathrm{LV}}-\eta_{\mathrm{TD}}\right) / \eta_{\mathrm{LV}} \times 100
$$

\section{Statistical Analysis}

Statistical analysis was performed using SPSS software (version 16.00 [SPSS Inc.] for Windows [Microsoft]). All numeric values are given as mean $\pm \mathrm{SD}$. Differences among the acquisitions concerning LV thickness, background variability, cavity contrast, and TD contrast were assessed by paired $t$ test and a 3-way factorial ANOVA. Reconstruction protocols, positioning combinations, and count statistics were considered as independent variables (factors) and image quality indices as dependent variables. All $P$ values less than 0.05 were considered to be statistically significant

\section{RESULTS}

\section{Qualitative Evaluation}

Images were qualitatively reviewed in random sequence, with operators masked to acquisition time and dose. Images acquired with $100 \%$ dose with very low counts (i.e., those acquired with an acquisition time of $1 \mathrm{~s} / \mathrm{view}$ ) showed severe inhomogeneity, with false defects in the anteroseptal and inferolateral walls. Images with an intermediate acquisition time (2-4 s/view) were more homogeneous but still exhibited discernible false defects. The interobserver concordance was excellent $(100 \%)$ both for images with very low count statistics and for images with longer acquisition times (i.e., 1, 2, 6, 8, and $12 \mathrm{~s} /$ view). Those images acquired with 3 and $4 \mathrm{~s}$ generated disagreements in 1 of the 3 operators, only concerning the evaluation of the small false-positive defects. Disagreement within the idealized confines of the phantom study would likely be amplified in clinical application. Therefore, we considered as adequate only images with very-good quality (i.e., those acquired with at least an acquisition time of $6 \mathrm{~s} / \mathrm{view}$, which did not show any false-positive defects) (Figs. 2-3).

The same approach was followed when evaluating images acquired with different doses. Very-good-quality images were acquired with at least $12 \mathrm{~s} /$ view for the $50 \%, 25 \%$, and $18 \%$ doses. No interobserver discrepancy was found for lower doses.

\section{Quantitative Evaluation}

To determine the effect of acquisition time on image quantification, analysis was performed at $100 \%$ dose over all acquisition times. Because qualitative evaluation for the less-than-100\% dose showed that only images acquired with at least $12 \mathrm{~s} / \mathrm{view}$ were of sufficient quality, we performed quantitative analysis only with this acquisition time for the $50 \%, 25 \%$, and $18 \%$ doses.

LV Wall Thickness. Table 1 shows FWHM values for all images, acquired with different doses and at different times. No statistically

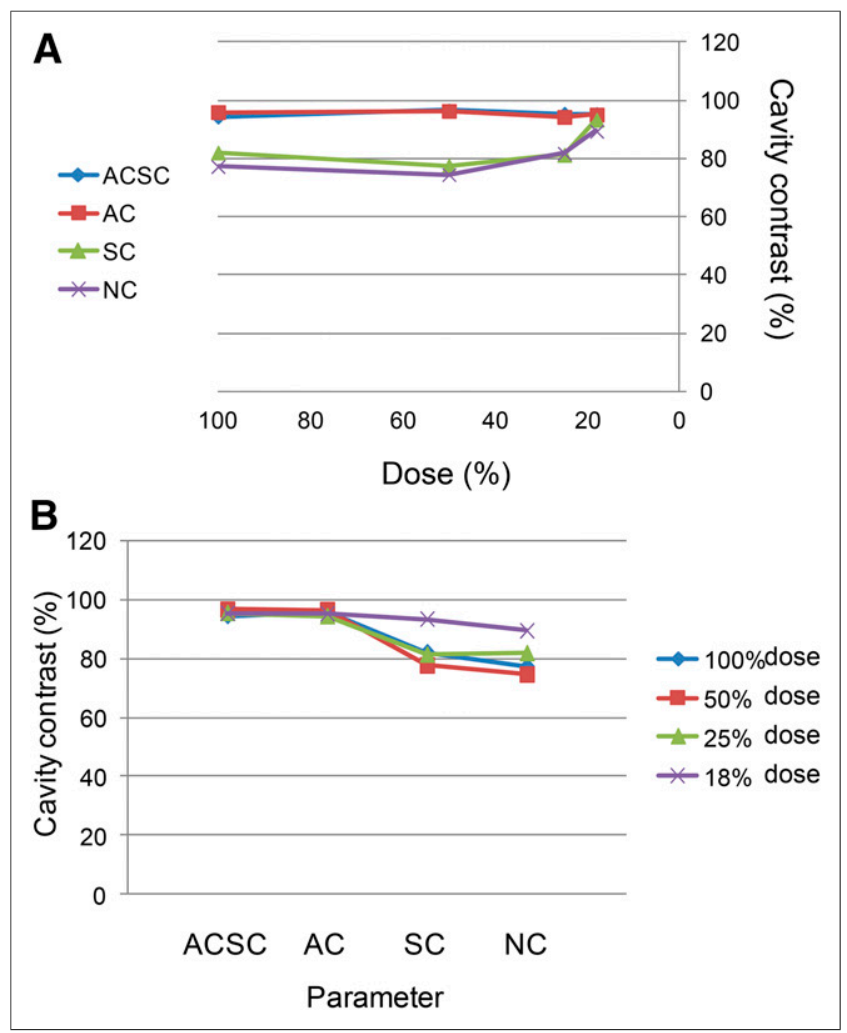

FIGURE 5. Cavity contrast with different applied reconstruction corrections across variable doses $(A)$ and with different doses across variable reconstruction corrections (B). Cavity contrast was consistent across different doses. Attenuation correction increased cavity contrast but did not reach statistical significance. For each dose, cavity contrast is constant when same correction is applied. 
TABLE 3

Total Counts and TD Contrast Values for Different Doses and Acquisition Times

\begin{tabular}{|c|c|c|c|c|c|}
\hline \multirow[b]{2}{*}{ s/view } & \multirow[b]{2}{*}{ Total counts $\left(\times 10^{5}\right)$} & \multicolumn{4}{|c|}{ TD contrast $(\%)$} \\
\hline & & ACSC & $A C$ & SC & NC \\
\hline \multicolumn{6}{|c|}{$100 \%$ dose } \\
\hline 1 & 1.1 & $72.4 \pm 3.9$ & $75.9 \pm 2.7$ & $58.7 \pm 2.7$ & $57.6 \pm 3.4$ \\
\hline 2 & 2.0 & $60.7 \pm 7.1$ & $77.1 \pm 4.0$ & $60.7 \pm 4.0$ & $59.9 \pm 3.1$ \\
\hline 3 & 3.0 & $67.2 \pm 3.1$ & $69.9 \pm 2.6$ & $58.7 \pm 2.3$ & $57.4 \pm 3.1$ \\
\hline 4 & 4.1 & $71.4 \pm 3.9$ & $71.0 \pm 3.3$ & $59.8 \pm 3.4$ & $59.9 \pm 2.7$ \\
\hline 6 & 5.9 & $66.6 \pm 4.9$ & $68.4 \pm 2.4$ & $56.7 \pm 4.3$ & $59.2 \pm 2.7$ \\
\hline 8 & 7.8 & $67.5 \pm 6.1$ & $72.1 \pm 2.0$ & $60.4 \pm 3.0$ & $60.7 \pm 2.7$ \\
\hline 12 & 12.0 & $68.4 \pm 4.4$ & $71.6 \pm 1.8$ & $58.4 \pm 2.6$ & $58.6 \pm 2.6$ \\
\hline \multicolumn{6}{|c|}{$50 \%$ dose } \\
\hline 6 & 3.5 & $54.9 \pm 4.7$ & $70.6 \pm 2.1$ & $58.9 \pm 2.8$ & $58.0 \pm 4.3$ \\
\hline 12 & 6.5 & $46.2 \pm 3.8$ & $78.2 \pm 2.4$ & $55.8 \pm 2.5$ & $55.9 \pm 2.3$ \\
\hline \multicolumn{6}{|c|}{$25 \%$ dose } \\
\hline 6 & 1.8 & $68.6 \pm 2.7$ & $80.0 \pm 3.0$ & $68.1 \pm 1.7$ & $65.9 \pm 2.9$ \\
\hline 12 & 3.2 & $68.8 \pm 1.7$ & $80.2 \pm 1.7$ & $57.2 \pm 2.1$ & $60.2 \pm 2.6$ \\
\hline \multicolumn{6}{|c|}{$18 \%$ dose } \\
\hline 6 & 1.4 & $48.2 \pm 5.0$ & $72.2 \pm 4.1$ & $55.4 \pm 4.4$ & $52.6 \pm 2.7$ \\
\hline 12 & 2.5 & $53.5 \pm 3.2$ & $69.7 \pm 3.0$ & $56.0 \pm 2.8$ & $56.5 \pm 2.7$ \\
\hline 24 & 5.0 & $52.8 \pm 2.0$ & $67.8 \pm 3.4$ & $55.0 \pm 4.0$ & $57.6 \pm 2.7$ \\
\hline
\end{tabular}

There are significant differences in AC images (TD contrast values are higher) and ACSC images (there is a fall in TD contrast for $50 \%$ dose) (Figs. 6 and 7). Values are mean \pm SD.

significant differences were evidenced among all acquisitions with different doses and different reconstruction corrections.

Cavity Contrast. Table 2 shows cavity contrast measurements for all images, acquired with different doses and at different times. Reducing acquisition time at a $100 \%$ dose did not significantly alter the cavity contrast measurements. Cavity contrast tended to be higher in images corrected for attenuation (AC and ACSC), though a statistical significance was not reached $(P=0.20)$ (Fig. 4).

Reducing the administered dose with a constant acquisition time also did not significantly alter cavity contrast. The nonsignificant tendency for higher contrast after AC and ACSC remained $(P=0.21)$ (Fig. 5).

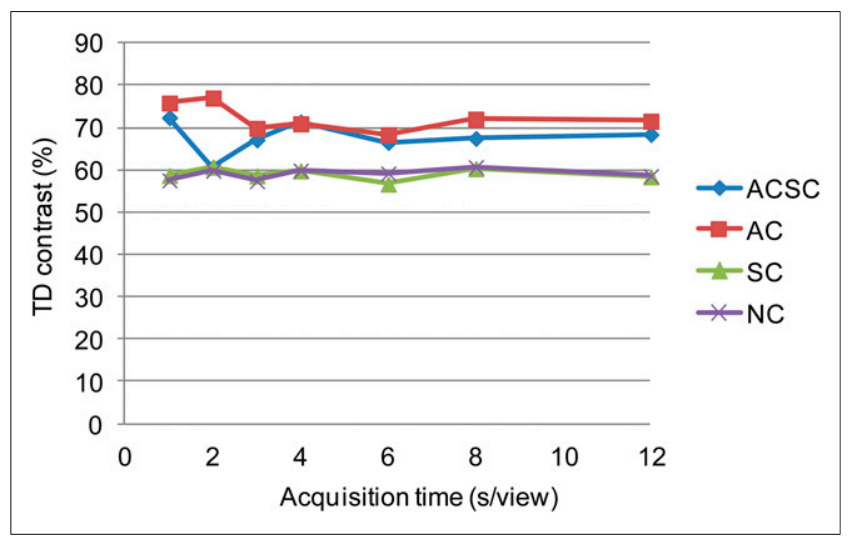

FIGURE 6. TD contrast at different acquisition times with constant dose (100\%). TD contrast remains unchanged for all acquisition times $(P=$ not significant).
At a $18 \%$ dose, cavity contrast did not differ between images acquired with either 12 or 24 s/view.

TD Contrast. Table 3 shows TD contrast measurements for all images, acquired with different doses and at different times. When acquisition time was reduced with a standard 100\% dose, TD contrast was unchanged. AC and ACSC images showed a modest increase in TD contrast, compared with uncorrected images $(P=$ 0.14) (Fig. 6). By contrast, lower administered dose affected the TD contrast, partially dependent on the corrections applied. Although in uncorrected and SC-alone images TD contrast was constant across doses, AC images exhibited significantly higher TD contrast $(P=0.04)$ at all doses. When both AC and SC were applied, TD contrast values were not statistically different, compared with $\mathrm{AC}, \mathrm{SC}$, and NC images $(P=0.16)$, but were inconsistent at variable doses. As shown in Figure 7, TD contrast was decreased at a $50 \%$ dose, restored somewhat at a $25 \%$ dose, and decreased again at a $18 \%$ dose. TD contrast values in ACSC images acquired with a $50 \%$ dose were significantly different from values in ACSC images obtained with other doses $(P=0.02)$. At a $18 \%$ dose, the reduction of ACSC image TD contrast approached statistical significance, compared with both $100 \%$ and $25 \%$ doses $(P=0.11)$.

\section{DISCUSSION}

This is the first study, to our knowledge, investigating the feasibility of a substantial dose reduction in MPI using the IQ SPECT protocol. We previously demonstrated an equivalence between quarter-time IQ SPECT and full-time standard SPECT with an ordered-subset expectation maximization reconstruction algorithm (4), but the impact of dose reduction has not been elucidated. 


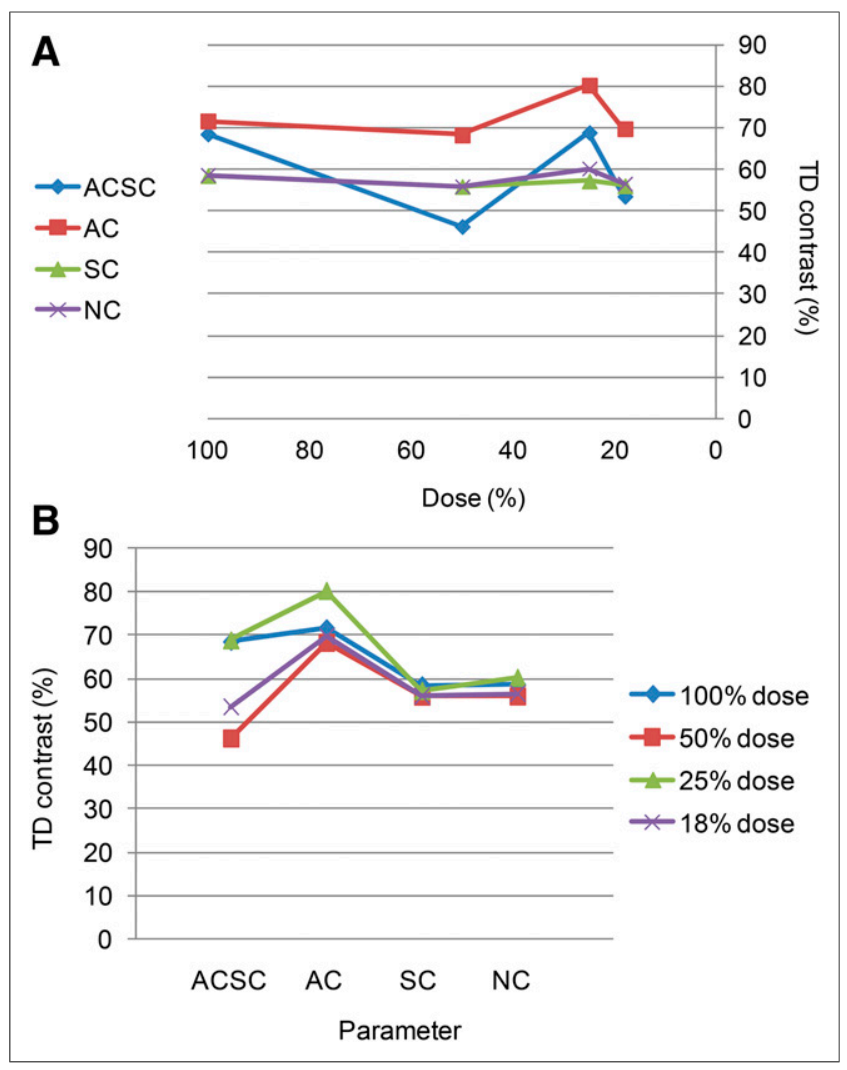

FIGURE 7. TD contrast with different applied reconstruction corrections across variable doses $(\mathrm{A})$ and with different doses across variable reconstruction corrections (B). TD contrast tended to be higher at $25 \%$ dose $(P=$ not significant). TD contrast was reduced in ACSC images for $50 \%$ dose with significant recovery at $25 \%(P<0.05)$.

Images obtained using a full standard dose showed no statistical difference in wall thickness or contrast ratio with lower acquisition times. The relative image quality was somewhat reduced on acquisitions shorter than 6 s/view, suggesting that, for a $740-\mathrm{MBq}$ standard dose, a minimum acquisition time of $6 \mathrm{~s} / \mathrm{view}$ is required.

When evaluating the performance of a package with a dedicated iterative reconstruction algorithm, reconstruction parameters (number of iterations, number of subsets, and postfilters) can have a significant impact on image quality (13). Moreover, integrated software to correct for attenuation and scatter can also lead to differences in the results (9). Therefore, while looking at the lowest possible dose, we also evaluated the impact of different corrections when performing MPI.

Wall thickness and contrast remained constant for $\mathrm{NC}, \mathrm{SC}$, and $\mathrm{AC}$ images across different doses, though $\mathrm{AC}$ appeared to improve contrast. Conversely, TD contrast in ACSC images was distinctly reduced with a $50 \%$ dose. Higher TD contrast with attenuation correction is a logical result and is consistent with more accurate estimation of TD extent as reported in the literature. Johansen et al. demonstrated a significant diagnostic modification in approximately $20 \%$ of abnormal studies when AC was applied, predominantly within the right coronary artery territory (14). Similar findings were reported by Giubbini et al. (15), in which AC significantly reduced both summed stress score and summed rest score; they reasoned that without AC the scar size was systematically overestimated.

These MPI studies, using parallel-hole collimators, report inferior and posterobasal wall artifacts due to diaphragmatic attenuation and the presence of typical apex thinning. When IQ SPECT is used, these same territories are affected, but the apparent size of such artifacts appears increased because of the different collimator shape $(4,7)$. Regional counts in these high-attenuation territories dramatically affected the TD contrast calculation in the absence of AC.

The unexpected drop in TD contrast with a $50 \%$ dose and relative recovery of the correction with further dose reductions requires further examination.

We may speculate that the particular collimation shape can influence the estimation of scatter and generation of attenuation maps, particularly with lower count statistics. The simultaneous application of 2 correction parameters would amplify such an error and contribute to a degradation of image quality (16). The recovery of TD contrast with further reduced administered doses may relate to the manufacturer default reconstruction algorithm settings, which may be more optimal for a quarter-dose protocol, compared with a $50 \%$ dose. As such, the algorithm may compensate more effectively for the count reduction in a specific dose range, manifesting as an improvement of image quality. With further dose reduction, the compensation tends to be less effective, as evidenced by reloss of TD contrast values at an $18 \%$ dose.

A previous study (9), using iterative reconstruction algorithms applied to standard systems with parallel-hole collimators, suggested that ACSC images were more robust than with AC alone. The difference from the present study may be explained by the difference in collimation and reconstruction software.

There is an apparent discrepancy in that reducing relative imaging time appears to have a more dramatic effect on image quality than a comparable reduction of injected dose. Dose reduction was always evaluated at $25 \%$ of standard acquisition time. At one eighth of the standard acquisition time (i.e., 6 s/view, one eighth of the standard scan), image quality begins to deteriorate, whereas a dose reduction to $18 \%$ of the standard dose at onequarter the standard acquisition time (i.e.,12 s/view, one twentyfourth of the standard scan) has a lesser effect on image quality. Part of this difference may derive from lost time due to camera movement and settling, as evidenced by more pronounced count reduction with lower time acquisition versus lower administered dose (i.e., 540 kcounts at 6 s/view vs. 650 kcounts at $50 \%$ dose, $12 \mathrm{~s} / \mathrm{view})$. With longer acquisitions, this dead time is likely negligible but is accentuated with shorter times per view. However, the resultant $17 \%-20 \%$ difference in total counts is insufficient to fully explain the discrepant effect on visual image quality. One may speculate that the uniformity of image reconstruction is more finely attuned to reduced global counts, but a complete explanation of this aberration requires further investigation.

Our study has some limitations. First, acquisitions with different times per view were sequentially performed; therefore, results could be theoretically influenced by ${ }^{99 \mathrm{~m}} \mathrm{Tc}$ decay. However, time interval between the first and the last examination within the same established dose was limited (maximum, $20 \mathrm{~min}$ ). Second, we did not evaluate the influence of other iterative reconstruction parameters (i.e., number of subsets, iterations, and resolution recovery) on the results. Third, these results were obtained in an ideal standard situation and might not be completely applicable in clinical practice; therefore, further clinical studies on patients are necessary to validate the clinical relevance of the findings. All acquisitions and data points derive from a single phantom source and activity loading. Finally, the phantom used in this study only allows the evaluation of ungated SPECT images; therefore, the results could be 
different when higher count statistics are required, as in the case of electrocardiography-gated studies, and the impact of motion cannot be evaluated.

In summary, an accurate evaluation was provided by acquiring a phantom MPI study with a standard dose and an acquisition time of 6 s/view (i.e., approximately one eighth, compared with the standard protocol). With regard to the administered dose, although as low as an $18 \%$ dose provided sufficient image quality, such a dramatic dose reduction may be excessive. For clinical application, many additional factors such as gated acquisitions, motion, and patient-induced scatter may complicate corrections and degrade image quality, and in vivo studies are warranted. Regarding reconstruction corrections, we would also suggest the use of noncorrected and AC images in clinical practice, because of less variability under different conditions.

Acquiring data of good quality with lower radioactivity would reduce patients' effective dose to approximately $2.5-3 \mathrm{mSv}$ for a stress-rest study with ${ }^{99 \mathrm{~m}} \mathrm{Tc}$-labeled tracers, much lower than current protocols with general-purpose low-energy and high-resolution collimator cameras $(\sim 9-10 \mathrm{mSv})$ and similar to those obtained with the new cadmium-zinc-telluride cameras (17). Moreover, the possibility to perform SPECT studies with reduced tracer activities may be helpful to overcome limitations due to ${ }^{99 \mathrm{~m}} \mathrm{Tc}$ shortages and could also address the requirement for population dose containment.

\section{CONCLUSION}

We demonstrated that IQ SPECT in a phantom study effectively preserved both image quality and quantitative contrast with reduced acquisition time or administered dose. If translated to clinical application, these protocols may improve patient comfort and reduce radioexposure without compromising diagnostic accuracy, which could be of particular interest for those centers in which a new dedicated heart camera system is not available.

\section{DISCLOSURE}

The costs of publication of this article were defrayed in part by payment of page charges. Therefore, and solely to indicate this fact, this article is hereby marked "advertisement" in accordance with 18 USC section 1734. No potential conflicts of interest relevant to this article are reported.

\section{REFERENCES}

1. Berman DS, Hachamovitch R, Shaw LJ, et al. Roles of nuclear cardiology, cardiac computed tomography, and cardiac magnetic resonance: assessment of patients with suspected coronary artery disease. J Nucl Med. 2006;47:74-82.

2. Einstein AJ, Blankstein R, Andrews H, et al. Comparison of image quality, myocardial perfusion, and left ventricular function between standard imaging and single-injection ultra-low-dose imaging using a high-efficiency SPECT camera: the MILLISIEVERT study. J Nucl Med. 2014;55:1430-1437.

3. DePuey E G. Advances in SPECT camera software and hardware: currently available and new on the horizon. $J$ Nucl Cardiol. 2012;19:551-581.

4. Caobelli F, Pizzocaro C, Paghera B, Guerra UP. Evaluation of patients with coronary artery disease: IQ-SPECT protocol in myocardial perfusion imagingpreliminary results. Nuklearmedizin. 2013;52:178-185.

5. Imbert L, Poussier S, Franken PR, et al. Compared performance of high-sensitivity cameras dedicated to myocardial perfusion SPECT: a comprehensive analysis of phantom and human images. J Nucl Med. 2012;53:1897-1903.

6. Rajaram R, Bhattacharya M, Ding $\mathrm{XH}$, et al. Tomographic performance characteristics of the IQ.SPECT system. IEEE Nucl Sci Symp Conf Rec. 2011: 2451-2456.

7. Vija H, Malmin R, Yahil A, Zeintl J, Bhattacharya M, Rempel TD. A method for improving the efficiency of myocardial perfusion imaging using conventional SPECT and SPECT/CT imaging systems. IEEE Nucl Sci Symp Conf Rec. 2010: 3433-3437.

8. Ali I, Ruddy TD, Almgrahi A, Anstett FG, Wells RG. Half-time SPECT myocardial perfusion imaging with attenuation correction. J Nucl Med. 2009;50: 554-562.

9. Zoccarato O, Scabbio C, De Ponti E, et al. Comparative analysis of iterative reconstruction algorithms with resolution recovery for cardiac SPECT studies: a multi-center phantom study. J Nucl Cardiol. 2014;21:135-148.

10. Higley B, Smith FW, Smith T, et al. Technetium-99m-1,2-bis[bis(2-ethoxyethyl) phosphino]ethane: human biodistribution, dosimetry and safety of a new myocardial perfusion imaging agent. J Nucl Med. 1993;34:30-38.

11. Hansen CL, Akinboboye OO, Berman DS, et al. Myocardial perfusion and function single photon emission computed tomography. J Nucl Cardiol. 2007;14: e39-e60.

12. Rasband WS. ImageJ. National Institutes of Health website. http://imagej.nih. gov/ij/. Accessed November 4, 2014.

13. Knoll P, Kotalova D, Köchle G, et al. Comparison of advanced iterative reconstruction methods for SPECT/CT. Z Med Phys. 2012;22:58-69.

14. Johansen A, Grupe P, Veje A, Nielsen Braad PE, Høilund-Carlsen PF. Scatter and attenuation correction changes interpretation of gated myocardial perfusion imaging. Eur J Nucl Med Mol Imaging. 2004;31:1152-1159.

15. Giubbini RM, Gabanelli S, Lucchini S, et al. The value of attenuation correction by hybrid SPECT/CT imaging on infarct size quantification in male patients with previous inferior myocardial infarct. Nucl Med Commun. 2011;32:1026-1032.

16. Caobelli F, Ren Kaiser S, Thackeray JT, et al. The importance of a correct positioning of the heart using IQ-SPECT system with multifocal collimators in myocardial perfusion imaging: a phantom study. J Nucl Cardiol. October 2, 2014 [Epub ahead of print].

17. Duvall WL, Croft LB, Godiwala T, Ginsberg E, George T, Henzlova MJ. Reduced isotope dose with rapid SPECT MPI imaging: initial experience with a CZT SPECT camera. J Nucl Cardiol. 2010;17:1009-1014. 